Proyecciones Journal of Mathematics

Vol. 32, No 4, pp. 393-408, December 2013.

Universidad Católica del Norte

Antofagasta - Chile

\title{
On generalized binomial series and strongly regular graphs
}

\author{
Vasco Moço Mano, Luís António de Almeida Vieira \\ University of Porto, Portugal \\ and \\ Enide Andrade Martins \\ University of Aveiro, Portugal \\ Received : April 2013. Accepted : August 2013
}

\begin{abstract}
We consider a strongly regular graph, $G$, and associate a three dimensional Euclidean Jordan algebra, $\mathcal{V}$, to its adjacency matrix A. Then, by considering binomial series of Hadamard powers of the idempotents of the unique complete system of orthogonal idempotents of $\mathcal{V}$ associated to A, we establish feasibility conditions for the existence of strongly regular graphs.
\end{abstract}

Keyword : Strongly regular graph Euclidean Jordan algebra Matrix analysis. 
394 Vasco Moço M., Luís A. de Almeida V. and Enide Andrade M.

\section{Introduction}

The concept of Euclidean Jordan algebra was introduced in 1934 by Pascual Jordan, John von Neumann and Eugene Wigner in the paper On an algebraic generalization of the quantum mechanical formalism [12]. This concept has had a wide range of applications. For instance, there are applications to the theory of statistics (see [17]), to interior point methods (see $[7,8]$ ) and to combinatorics (see [4]). Detailed literature on Euclidean Jordan algebras can be found in the monograph by Faraut and Korányi, [6].

Strongly regular graphs are a class of graphs introduced in 1963 in a paper by R. C. Bose, entitled Strongly regular graphs, partial geometries and partially balanced designs, [2]. These graphs are defined by a set of parameters that must satisfy several feasibility conditions. The Krein conditions and the absolute bounds (see, for instance, [9]) are among the most used admissibility inequalities. For detailed information on strongly regular graphs, the reader may consult the following references: $[1,3,9$, $11,14,18]$.

In this work we explore the relationship between Euclidean Jordan algebras and strongly regular graphs, in order to find feasibility conditions for the existence of strongly regular graphs.

In the present work we consider a strongly regular graph, $G$, and associate a three dimensional Euclidean Jordan algebra, $\mathcal{V}$, to its adjacency matrix $A$. Then, by considering binomial series of Hadamard powers of the idempotents of the unique complete system of orthogonal idempotents of $\mathcal{V}$ associated to $A$, we establish feasibility conditions for the existence of strongly regular graphs. These admissibility conditions are generalizations of the conditions obtained in the extended abstract [15] and in the paper [16]. Instead of using geometric series with natural exponents as it was done in $[15,16]$, we now consider binomial series with positive real exponents and we also apply our conclusions to the complement graph $\bar{G}$.

Euclidean Jordan algebras are briefly introduced in Section 2, while in Section 3 the theory of strongly regular graphs is surveyed. Then, in Section 4 , by constructing a special series of Hadamard powers of a particular idempotent of $\mathcal{V}$, and applying some matrix techniques, we establish a feasibility condition for the existence of strongly regular graphs. Finally by observing the relationship between a strongly regular graph and its complement, we extract further conclusions for parameter sets with $k<n / 2$ and $k>n / 2-1$. We finish the paper with some experimental results that 
confirm our conclusions (Section 5).

\section{Euclidean Jordan algebras}

In this section we introduce the most important definitions and results about power-associative algebras and Euclidean Jordan algebras. Adittional literature can be found in the monograph by Faraut and Korányi, [6], and in Koecher's lecture notes, [13].

Let $F$ be the field $\mathbf{R}$ or $\mathbf{C}$ and $A$ be a $n$-dimensional algebra over $F$ with the bilinear mapping $(x, y) \mapsto x \cdot y$ with the unit element e. The algebra $\mathcal{A}$ is power-associative if for any $x$ in $\mathcal{A}$ the algebra generated by $x$ and $\mathbf{e}$ is associative.

For $x$ in $\mathcal{A}$, the rank of $x$ is the least natural number $k$ such that $\left\{\mathbf{e}, \mathbf{x}, \ldots, \mathbf{x}^{\mathbf{k}}\right\}$ is linearly dependent and we write $\operatorname{rank}(x)=k$. Since for all $x$ in $\mathcal{A}$ we have $\operatorname{rank}(x) \leq n$, we define the $\operatorname{rank}$ of $\mathcal{A}$ as being the natural number $\operatorname{rank}(\mathcal{A})=\max \{\operatorname{rank}(x): x \in \mathcal{A}\}$.

An element $x$ in $A$ is regular if $\operatorname{rank}(x)=\operatorname{rank}(\mathcal{A})$. Let $x$ be a regular element of $A$ and $r=\operatorname{rank}(x)$.

Then, there exist polynomials $a_{1}, a_{2}, \ldots, a_{r-1}$ and $a_{r}$ on $\mathcal{A}$, not all being zero, such that

$$
x^{r}-a_{1}(x) x^{r-1}+\cdots+(-1)^{r} a_{r}(x) \mathbf{e}=0,
$$

where 0 is the null vector of $\mathcal{A}$ and each $a_{j}(x)$ is a homogeneous polynomial of degree $j$. Taking into account (2.1) we conclude that the polynomial

$$
p(x, \lambda)=\lambda^{r}-a_{1}(x) \lambda^{r-1}+\cdots+(-1)^{r} a_{r}(x)
$$

is the minimal polynomial of $x$. When $x$ is non regular the minimal polynomial of $x$ has degree less than $r$. We call the roots of the minimal polynomial of $x$ the eigenvalues of $x$.

The real vector space of real symmetric matrices of order $n, \mathcal{V}=$ $\operatorname{Sym}(n, \mathbf{R})$, equipped with the bilinear map $x \bullet y=(x y+y x) / 2$, with $x, y$ in $\mathcal{V}$, is a real power-associative algebra whose unit is $\mathbf{e}=I_{n}$.

A Jordan algebra $\mathcal{A}$ over $\mathcal{F}$ is a vector space over the field $F$ with a bilinear map $(x, y) \mapsto x \cdot y$, such that for all $x$ and $y$ in $\mathcal{A}$ we have

(i) $x \cdot y=y \cdot x$,

(ii) $x \cdot\left(x^{2} \cdot y\right)=x^{2} \cdot(x \cdot y)$, 
396 Vasco Moço M., Luís A. de Almeida V. and Enide Andrade M.

where $x^{2}=x \cdot x$.

Let $\mathcal{A}$ be a finite dimensional associative algebra over the field $\mathcal{F}$ with the bilinear map $(x, y) \mapsto x \cdot y$. We introduce on $\mathcal{A}$ a structure of Jordan algebra by considering a new product $\bullet$ defined by $x \bullet y=(x \cdot y+y \cdot x) / 2$, for all $x$ and $y$ in $\mathcal{A}$. This product is called the Jordan product.

The real vector space $\mathcal{V}=\operatorname{Sym}(n, \mathbf{R})$ is a real Jordan algebra when endowed with the bilinear map $\bullet$ given by $x \bullet y=(x y+y x) / 2$ for all $x$ and $y$ in $\mathcal{V}$, where $x y$ is the usual matrix multiplication of $x$ and $y$.

From now on, a Jordan algebra $\mathcal{A}$ is always a finite dimensional algebra over the field $\mathcal{F}$ with unit element e. If $A$ is a Jordan algebra then $\mathcal{A}$ is power-associative.

An Euclidean Jordan algebra $\mathcal{A}$ is a Jordan algebra with an inner product $\langle\cdot, \cdot\rangle$ such that

$$
<x \cdot y, z>=\langle y, x \cdot z\rangle
$$

for all $x, y$ and $z$ in $\mathcal{A}$.

The real vector space $\mathcal{V}=\operatorname{Sym}(n, \mathbf{R})$ is a real Euclidean Jordan algebra when endowed with the Jordan product and with the inner product < $x, y>=\operatorname{tr}(x y)$, where tr denotes the usual trace of matrices.

Let $A$ be a real Euclidean Jordan algebra with unit element e. An element $c$ in $\mathcal{A}$ is an idempotent if $c^{2}=c$. Two idempotents $c$ and $d$ in $\mathcal{A}$ are orthogonal if $c \cdot d=0$. The set $\left\{c_{1}, c_{2}, \ldots, c_{l}\right\}$ is a complete system of orthogonal idempotents if

(i) $c_{i}^{2}=c_{i}$, for $i=1, \ldots, l$,

(ii) $c_{i} \cdot c_{j}=0$, if $i \neq j$,

(iii) $\quad \sum_{i=1}^{l} c_{i}=\mathbf{e}$.

An idempotent $c$ is primitive if it is a nonzero idempotent of $\mathcal{A}$ and if it cannot be written as a sum of two nonzero idempotents. We say that $\left\{c_{1}, c_{2}, \ldots, c_{k}\right\}$ is a Jordan frame if $\left\{c_{1}, c_{2}, \ldots, c_{k}\right\}$ is a complete system of orthogonal idempotents such that each idempotent is primitive.

Theorem 1. ([6], pg. 43).

Let $A$ be a real Euclidean Jordan algebra. Then for $x$ in $A$ there exist unique real numbers $\lambda_{1}, \lambda_{2}, \ldots, \lambda_{k}$, all distinct, and a unique complete system of orthogonal idempotents $\left\{c_{1}, c_{2}, \ldots, c_{k}\right\}$ such that

$$
x=\lambda_{1} c_{1}+\lambda_{2} c_{2}+\cdots+\lambda_{k} c_{k} .
$$


The numbers $\lambda_{j}$ of (2.4) are the eigenvalues of $x$ and the decomposition (2.4) is the spectral decomposition of $x$. If $x$ is an element of a real Euclidean Jordan algebra $\mathcal{A}$ with spectral decomposition $x=\lambda_{1} c_{1}+\lambda_{2} c_{2}+$ $\cdots+\lambda_{k} c_{k}$, then the minimal polynomial of $x$ is the polynomial $p$ such that

$$
p(x, \lambda)=\Pi_{i=1}^{k}\left(\lambda-\lambda_{i}\right) .
$$

\section{Strongly regular graphs}

Throughout this text a graph $G$ is a pair $(V(G), E(G))$ of a vertex set, $V(G)$, and an edge set $E(G)$, where an edge is an unordered pair of distinct vertices of $G$. An edge whose endpoints are the vertices $u$ and $v$ is denoted by $u v$ and, in such a case, the vertices $u$ and $v$ are adjacent or neighbors. The number of vertices of $G,|V(G)|$, is called the order of $G$. A graph in which all pairs of vertices are adjacent (non-adjacent) is called a complete (null) graph. The number of neighbors of a vertex $v$ in $V(G)$ is called the degree of $v$. If all vertices of a graph $G$ have degree $k$, for some natural number $k$, then $G$ is $k$-regular. Along this paper we only consider simple graphs, that is, graphs with no loops (edges connected at both ends to the same vertex) and no more than one edge between any two different vertices.

We associate to $G$ an $n \times n$ matrix $A=\left[a_{i j}\right]$, where each $a_{i j}=1$, if $v_{i} v_{j} \in E(G)$, otherwise $a_{i j}=0$, called the adjacency matrix of $G$. The eigenvalues of $A$ are simply called the eigenvalues of $G$.

A simple, non-null and not complete graph $G$ is strongly regular with parameters $(n, k, a, c)$ if

1) $G$ is $k$-regular;

2) each pair of adjacent vertices has $a$ common neighbors;

3) each pair of non-adjacent vertices have $c$ common neighbors.

If $A$ is the adjacency matrix of a $(n, k, a, c)$-strongly regular graph $G$, then conditions 1) -3 ) are equivalent to

$$
\begin{aligned}
A J_{n} & =k J_{n}, \\
A^{2} & =k I_{n}+a A+c\left(J_{n}-A-I_{n}\right),
\end{aligned}
$$

where $I_{n}$ and $J_{n}$ denote the identity and the all one matrices of order $n$, respectively. The parameters of a $(n, k, a, c)$-strongly regular graph are not independent and are related by the equality

$$
k(k-a-1)=(n-k-1) c .
$$


It is well known (see, for instance, [9]) that if $G$ is a $(n, k, a, c)$-strongly regular graph, then it's complement $\bar{G}$ is a $(n, \bar{k}, \bar{a}, \bar{c})$-strongly regular graph, where

$$
\begin{aligned}
& \bar{k}=n-k-1, \\
& \bar{a}=n-2-2 k+c, \\
& \bar{c}=n-2 k+a .
\end{aligned}
$$

In order to exclude trivial cases we shall consider that $G$ and its complement are connected and therefore we have $0<c<k<n-1$. Note that while (3.2) and (3.4) produce positive numbers, the positivity of (3.3) is not guaranteed. Also, the eigenvalues of a $(n, k, a, c)$-strongly regular graph $G$ are $k, \theta$ and $\tau$, where $\theta$ and $\tau$ are given by

$$
\begin{aligned}
\theta & =\frac{a-c+\sqrt{(a-c)^{2}+4(k-c)}}{2}, \\
\tau & =\frac{a-c-\sqrt{(a-c)^{2}+4(k-c)}}{2} .
\end{aligned}
$$

Note that $\theta$ is positive and $\tau$ is negative. The eigenvalues of a strongly regular graph satisfy the following inequalities known as the Krein conditions, obtained in [19]:

$$
\begin{aligned}
& (\theta+1)(k+\theta+2 \theta \tau) \leq(k+\theta)(\tau+1)^{2}, \\
& (\tau+1)(k+\tau+2 \theta \tau) \leq(k+\tau)(\theta+1)^{2} .
\end{aligned}
$$

The multiplicities of the eigenvalues of a strongly regular graph can also be obtained as follows (see, for instance, [14]):

$$
\begin{aligned}
f & =\frac{1}{2}\left(n-1-\frac{(\theta+\tau)(n-1)+2 k}{\theta-\tau}\right), \\
g & =\frac{1}{2}\left(n-1+\frac{(\theta+\tau)(n-1)+2 k}{\theta-\tau}\right) .
\end{aligned}
$$

Regard that the formulae (3.9) and (3.10) must yield natural numbers. Furthermore, it was proven (see [5]) that $f$ and $g$ must also satisfy the following Seidel's absolute bounds:

$$
\begin{aligned}
& n \leq \frac{f(f+3)}{2}, \\
& n \leq \frac{g(g+3)}{2} .
\end{aligned}
$$


Besides the conditions presented above, A. E. Brouwer improved a result from Neumaier (see [18, Theorem 4.7]) and obtained the following condition (see [3]), known as the claw bound:

Theorem 2 ([14], Theorem 21.7). Let $G$ be a $(n, k, a, c)$-strongly regular graph, such that $0<c<k<n-1$, whose adjacency matrix has the eigenvalues $k, \theta$ and $\tau$. If $c \neq \tau^{2}$ and $c \neq \tau(\tau+1)$, then

$$
2(\theta+1) \leq \tau(\tau+1)(c+1)
$$

A parameter set $(n, k, a, c)$ for which (3.3), (3.9) and (3.10) produce positive integers and that also satisfies equality (3.1) and inequalities (3.7), (3.8), (3.11), (3.12) and (3.13) is usually called a feasible set and all the conditions above are called feasibility conditions. With these feasibility conditions there are many parameter sets that are excluded as possible strongly regular graphs. However, there are still many parameter sets for which we do not know if they correspond to a strongly regular graph. In this work we deduce conditions to claim the unfeasibility of certain parameter sets of strongly regular graphs.

\section{Feasibility conditions for strongly regular graphs}

From now on we consider the Euclidean Jordan algebra of real symmetric matrices of order $n, \mathcal{V}=\operatorname{Sym}(n, \mathbf{R})$, endowed with the Jordan product already defined and the inner product defined for matrices $A, B$ in $\mathcal{V}$ as $<A, B>=\operatorname{tr}(A B)$, where $\operatorname{tr}$ is the classical trace of matrices, that is the sum of its eigenvalues.

Let $G$ be a $(n, k, a, c)$-strongly regular graph such that $0<c<k<n-1$, and let $A$ be the adjacency matrix of $G$ with three distinct eigenvalues, namely the degree of regularity $k$, and the restricted eigenvalues $\theta$ and $\tau$, given in (3.5) and (3.6). Recall that $k$ and $\theta$ are the positive eigenvalues and $\tau$ is the negative eigenvalue of $A$. Now we consider the Euclidean Jordan subalgebra of $\mathcal{V}, \mathcal{V}^{\prime}$, spanned by $I_{n}$ and the powers of $A$. Since $A$ has three distinct eigenvalues, then $\mathcal{V}^{\prime}$ is a three dimensional Euclidean Jordan algebra with $\operatorname{rank}\left(\mathcal{V}^{\prime}\right)=3$.

Let $\mathcal{B}=\left\{E_{0}, E_{1}, E_{2}\right\}$ be the unique complete system of orthogonal idempotents of $\mathcal{V}$ associated to $A$, with

$$
E_{0}=\frac{1}{n} I_{n}+\frac{1}{n} A+\frac{1}{n}\left(J_{n}-A-I_{n}\right)=\frac{1}{n} J_{n},
$$


400 Vasco Moço M., Luís A. de Almeida V. and Enide Andrade M.

$$
\begin{aligned}
& E_{1}=\frac{-\tau n+\tau-k}{n(\theta-\tau)} I_{n}+\frac{n+\tau-k}{n(\theta-\tau)} A+\frac{\tau-k}{n(\theta-\tau)}\left(J_{n}-A-I_{n}\right), \\
& E_{2}=\frac{\theta n+k-\theta}{n(\theta-\tau)} I_{n}+\frac{-n+k-\theta}{n(\theta-\tau)} A+\frac{k-\theta}{n(\theta-\tau)}\left(J_{n}-A-I_{n}\right),
\end{aligned}
$$

We now introduce a more specific notation. Let $p$ be a nonnegative integer and denote by $M_{n}(\mathbf{R})$ the set of square matrices of order $n$ with real entries. For $B$ in $M_{n}(\mathbf{R})$, we denote by $B^{\circ p}$ and $B^{\otimes p}$ the Hadamard power and the Kronecker power of order $p$ of $B$, respectively, with $B^{\circ 1}=B$, $B^{\circ 0}=J_{n}$ and $B^{\otimes 1}=B$. We consider $E_{j}^{\circ i}=\left(E_{j}\right)^{\circ i}$ and $E_{j}^{\otimes i}=\left(E_{j}\right)^{\otimes i}$ for all natural numbers $i$ and $j$ such that $0 \leq j \leq 2$ and $i \geq 0$.

Consider the following spectral decomposition of $A, A=k E_{0}+\theta E_{1}+$ $\tau E_{2}$. Let $l$ in $\mathbf{N}, \alpha$ in $\mathbf{R}^{+}$and $S_{(2 l) \alpha}^{\otimes}$ be the following sum:

$$
\begin{aligned}
& S_{(2 l) \alpha}^{\otimes}=\left(\begin{array}{c}
-\alpha \\
0
\end{array}\right) J_{n}^{\otimes 2 l}-\left(\begin{array}{c}
-\alpha \\
1
\end{array}\right) J_{n}^{\otimes 2 l-2} \otimes E_{2}^{\otimes 2}+\left(\begin{array}{c}
-\alpha \\
2
\end{array}\right) J_{n}^{\otimes 2 l-4} \otimes E_{2}^{\otimes 4}+\cdots \\
& (4.1)+(-1)^{l-1}\left(\begin{array}{c}
-\alpha \\
l-1
\end{array}\right) J_{n}^{\otimes 2} \otimes E_{2}^{\otimes 2 l-2}+(-1)^{l}\left(\begin{array}{c}
-\alpha \\
l
\end{array}\right) E_{2}^{\otimes 2 l} .
\end{aligned}
$$

where each summand is a Kronecker product with $2 l$ factors. Recall that for any real number $\alpha$ and each nonegative integer $k \geq 1$,

$$
\left(\begin{array}{l}
\alpha \\
k
\end{array}\right)=\frac{\alpha(\alpha-1) \cdots(\alpha-k+1)}{k !},
$$

with $\left(\begin{array}{l}\alpha \\ 0\end{array}\right)=1$, that is the generalized binomial number. The sum $S_{(2 l) \alpha}^{\otimes}$ has a principal submatrix given by:

$$
\begin{aligned}
S_{(2 l) \alpha}^{\circ} & =\left(\begin{array}{c}
-\alpha \\
0
\end{array}\right) J_{n}^{\circ 2 l}-\left(\begin{array}{c}
-\alpha \\
1
\end{array}\right) J_{n}^{\circ 2 l-2} \circ E_{2}^{\circ 2}+\left(\begin{array}{c}
-\alpha \\
2
\end{array}\right) J_{n}^{\circ 2 l-4} \circ E_{2}^{\circ 4}+\cdots \\
4.2)+ & +(-1)^{l-1}\left(\begin{array}{c}
-\alpha \\
l-1
\end{array}\right) J_{n}^{\circ 2} \circ E_{2}^{\circ 2 l-2}+(-1)^{l}\left(\begin{array}{c}
-\alpha \\
l
\end{array}\right) E_{2}^{\circ 2 l} .
\end{aligned}
$$

Observe that $S_{(2 l) \alpha}^{\circ}=\sum_{i=0}^{l}(-1)^{i}\left(\begin{array}{c}-\alpha \\ i\end{array}\right) E_{2}^{\circ 2 i}$. Let $q_{(2 l) \alpha}^{0}, q_{(2 l) \alpha}^{1}$ and $q_{(2 l) \alpha}^{2}$ be the real numbers such that $S_{(2 l) \alpha}^{\circ}=\sum_{i=0}^{2} q_{(2 l) \alpha}^{i} E_{i}$. Since the set

$$
C=\left\{E_{i_{1}} \otimes E_{i_{2}} \otimes \cdots \otimes E_{i_{2 l}}: i_{1}, i_{2}, \ldots, i_{2 l} \in\{0,1,2\}\right\}
$$

is a complete system of orthogonal idempotents that is a basis of the real Euclidean Jordan subalgebra of $\operatorname{Sym}\left(n^{2 l}, \mathbf{R}\right), \mathcal{V}^{\otimes 2 l}$, spanned by $I_{n}^{\otimes 2 l}$ and 
the natural powers of $A^{\otimes 2 l}$, then the minimal polynomial of $S_{(2 l) \alpha}^{\otimes}$ is

$$
p_{\alpha}(\lambda)=(\lambda-0) \prod_{i=0}^{l}\left(\lambda-(-1)^{i}\left(\begin{array}{c}
-\alpha \\
i
\end{array}\right) n^{2(l-i)}\right) .
$$

Note that to obtain the minimal polynomial we use (2.5) and the system of orthogonal idempotents, $\mathcal{C}$, in each summand of (4.1).

Attending that the matrix in (4.2) is a principal submatrix of $S_{(2 l) \alpha}^{\otimes}$ and $p_{\alpha}$ is the minimal polynomial of $S_{(2 l) \alpha}^{\otimes}$. By the interlacing theorem (see [10, Theorem 4.3.15]), its eigenvalues are all nonnegative. Regarding that

$$
\begin{aligned}
S_{(2 l) \alpha}^{\circ} & =\sum_{i=0}^{l}(-1)^{i}\left(\begin{array}{c}
-\alpha \\
i
\end{array}\right)\left(\frac{\theta n+k-\theta}{n(\theta-\tau)}\right)^{2 i} I_{n} \\
& +\sum_{i=0}^{l}(-1)^{i}\left(\begin{array}{c}
-\alpha \\
i
\end{array}\right)\left(\frac{-n+k-\theta}{n(\theta-\tau)}\right)^{2 i} A \\
& +\sum_{i=1}^{l}(-1)^{i}\left(\begin{array}{c}
-\alpha \\
i
\end{array}\right)\left(\frac{k-\theta}{n(\theta-\tau)}\right)^{2 i}\left(J_{n}-A-I_{n}\right),
\end{aligned}
$$

since $|\tau|>1$, then

$$
\left|\frac{\theta n+k-\theta}{n(\theta-\tau)}\right|<1,\left|\frac{-n+k-\theta}{n(\theta-\tau)}\right|<1 \text { and }\left|\frac{k-\theta}{n(\theta-\tau)}\right|<1,
$$

and therefore the series $\sum_{i=0}^{+\infty}(-1)^{i}\left(\begin{array}{c}-\alpha \\ i\end{array}\right) E_{2}^{\circ 2 i}$ is convergent with sum $s_{\alpha}$.

The next theorem contains a new inequality for which the parameters of a strongly regular graph must satisfy.

Theorem 3. Let $G$ be a $(n, k, a, c)$-strongly regular graph, such that $0<$ $c<k<n-1$, whose adjacency matrix has the eigenvalues $k, \theta$ and $\tau$. Then, for any positive real number $\alpha$,

$$
\begin{aligned}
0 & \leq \frac{1}{\left(1-\left(\frac{\theta n+k-\theta}{n(\theta-\tau)}\right)^{2}\right)^{\alpha}}+\frac{1}{\left(1-\left(\frac{-n+k-\theta}{n(\theta-\tau)}\right)^{2}\right)^{\alpha}} \tau \\
& +\frac{1}{\left(1-\left(\frac{k-\theta}{n(\theta-\tau)}\right)^{2}\right)^{\alpha}}(-\tau-1) .
\end{aligned}
$$


402 Vasco Moço M., Luís A. de Almeida V. and Enide Andrade M.

Consider the real numbers $q_{\infty \alpha}^{0}, q_{\infty \alpha}^{1}, q_{\infty \alpha}^{2}$ such that

$$
s_{\alpha}=\lim _{l \rightarrow+\infty} S_{(2 l) \alpha}^{\circ}=q_{\infty \alpha}^{0} E_{0}+q_{\infty \alpha}^{1} E_{1}+q_{\infty \alpha}^{2} E_{2} .
$$

As

$$
S_{(2 l) \alpha}^{\circ}=q_{(2 l) \alpha}^{0} E_{0}+q_{(2 l) \alpha}^{1} E_{1}+q_{(2 l) \alpha}^{2} E_{2},
$$

applying limits to (4.6) and comparing expressions (4.5) and (4.6) we obtain $q_{\infty \alpha}^{0}=\lim _{l \rightarrow \infty} q_{(2 l) \alpha}^{0}, q_{\infty \alpha}^{1}=\lim _{l \rightarrow \infty} q_{(2 l) \alpha}^{1}, q_{\infty \alpha}^{2}=\lim _{l \rightarrow \infty} q_{(2 l) \alpha}^{2}$.

As the eigenvalues of $S_{(2 l) \alpha}^{\circ}$ are nonnegative, it follows that $q_{\infty \alpha}^{0} \geq 0$, $q_{\infty \alpha}^{1} \geq 0$ and $q_{\infty \alpha}^{2} \geq 0$. Then from identity (4.3) and doing some algebraic manipulations we obtain:

$$
q_{\infty \alpha}^{2}=\frac{1}{\left(1-\left(\frac{\theta n+k-\theta}{n(\theta-\tau)}\right)^{2}\right)^{\alpha}}+\frac{\tau}{\left(1-\left(\frac{-n+k-\theta}{n(\theta-\tau)}\right)^{2}\right)^{\alpha}}+\frac{-\tau-1}{\left(1-\left(\frac{k-\theta}{n(\theta-\tau)}\right)^{2}\right)^{\alpha}}
$$

thus proving our assertion.

Note that the other real numbers $q_{\infty \alpha}^{0}$ and $q_{\infty \alpha}^{1}$ are obtained with similar arguments. Inequality (4.4) from Theorem 3 allow us to deduce the next result for parameter sets with $k<n / 2$.

Corollary 1. Let $G$ be a $(n, k, a, c)$-strongly regular graph, such that $0<$ $c<k<n-1$, whose adjacency matrix has the eigenvalues $k, \theta$ and $\tau$. If $k<n / 2$, then

$-\tau(+\mathbb{Q} . \pi)-1)(4 \theta-2 \tau+1) \leq \frac{2 n}{n-2(k-\theta)} \theta(\theta+1)(2 \theta-2 \tau-1)(\theta-\tau+1)$.

From inequality (4.4) of Theorem 3, with $\alpha=1$, since $(-n+k-\theta)^{2}=$ $(n-k+\theta)^{2}$, one concludes that

$$
0 \leq \frac{1}{1-\left(\frac{\theta n+k-\theta}{n(\theta-\tau)}\right)^{2}}+\frac{1}{1-\left(\frac{n-k+\theta}{n(\theta-\tau)}\right)^{2}} \tau+\frac{1}{1-\left(\frac{k-\theta}{n(\theta-\tau)}\right)^{2}}(-\tau-1) .
$$

Associating terms in $\tau$ we obtain

$$
0 \leq \frac{\left(\frac{\theta n+k-\theta}{n(\theta-\tau)}\right)^{2}-\left(\frac{k-\theta}{n(\theta-\tau)}\right)^{2}}{\left[1-\left(\frac{\theta n+k-\theta}{n(\theta-\tau)}\right)^{2}\right]\left[1-\left(\frac{k-\theta}{n(\theta-\tau)}\right)^{2}\right]}+\frac{\left(\frac{n-k+\theta}{n(\theta-\tau)}\right)^{2}-\left(\frac{k-\theta}{n(\theta-\tau)}\right)^{2}}{\left[1-\left(\frac{n-k+\theta}{n(\theta-\tau)}\right)^{2}\right]\left[1-\left(\frac{k-\theta}{n(\theta-\tau)}\right)^{2}\right]} \tau .
$$



we get

Multiplying by $1-\left(\frac{k-\theta}{n(\theta-\tau)}\right)^{2}$ and after some algebraic manipulations

$$
0 \leq \frac{\frac{\theta(\theta n+2 k-2 \theta)}{n(\theta-\tau)^{2}}}{1-\left(\frac{\theta n+k-\theta}{n(\theta-\tau)}\right)^{2}}+\frac{\frac{n-2 k+2 \theta}{n(\theta-\tau)^{2}}}{1-\left(\frac{n-k+\theta}{n(\theta-\tau)}\right)^{2}} \tau .
$$

Multiplying by $(\theta-\tau)^{2}$, considering $k<n / 2$ and neglecting $-2 \theta$ we obtain

$$
0<\frac{\theta(\theta+1)}{1-\left(\frac{\theta n+k-\theta}{n(\theta-\tau)}\right)^{2}}+\frac{\frac{n-2 k+2 \theta}{n}}{1-\left(\frac{n-k+\theta}{n(\theta-\tau)}\right)^{2}} \tau .
$$

Now we will attend to the denominators of the right hand side of the inequality above. For the first one we will use the fact that $k<n / 2$, neglect $-\theta$ and simplify. As for the second denominator we will simplify using the difference of two squares and, along the way, neglect $-\theta$ and $-k+\theta$ :

$$
0<\frac{\theta(\theta+1)}{\frac{(-2 \tau-1)(4 \theta-2 \tau+1)}{4(\theta-\tau)^{2}}}+\frac{\frac{n-2 k+2 \theta}{n}}{\frac{\left(\theta-\tau-\frac{1}{2}\right)(\theta-\tau+1)}{(\theta-\tau)^{2}}} \tau .
$$

Finally, multiplying both terms of the inequality by $\frac{1}{4(\theta-\tau)^{2}}$,

$$
0<\frac{\theta(\theta+1)}{(-2 \tau-1)(4 \theta-2 \tau+1)}+\frac{n-2 k+2 \theta}{2 n(2 \theta-2 \tau-1)(\theta-\tau+1)} \tau,
$$

which can be rewritten as (4.7).

For a fixed $n, k$ and $\theta$ and analyzing inequality (4.7) we observe that the left hand side is a polynomial in $|\tau|$ of degree 3 and the right hand side is a polynomial in $|\tau|$ of degree 2 , both with positive leading coefficients. Therefore one may conclude that if $|\tau|$ is bigger than $\theta$, then $|\tau|$ cannot be too large relatively to the value of $\theta$.

Applying Corollary 1 to the complement graph, $\bar{G}$, we deduce the next result, that presents an inequality for graphs which satisfy $k>n / 2-1$.

Corollary 2. Let $G$ be a $(n, k, a, c)$-strongly regular graph, such that $0<$ $c<k<n-1$, whose adjacency matrix has the eigenvalues $k, \theta$ and $\tau$. If $k>n / 2-1$, then

$(\theta-1)(2 \theta-3)(-4 \tau+2 \theta+3)<\frac{2 n(-\tau+1)(-\tau+2)(-2 \tau+2 \theta-1)(\theta-\tau+1)}{2(k-\tau+2)-n}$. 
404 Vasco Moço M., Luís A. de Almeida V. and Enide Andrade M.

Regard that the condition $k<n / 2$ is equivalent to $\bar{k}>n / 2-1$. Therefore, applying inequality (4.7) of Theorem 1 to the parameter set $(n, \bar{k}, \bar{a}, \bar{c})$ already introduced by (3.2)-(3.4), of $\bar{G}$, the inequality (4.8) follows directly.

This result can be interpreted in a similar way than the previous one. For a fixed $n, k$ and $\tau$, inequality (4.8) presents a polynomial in $\theta$ of degree 3 on the left hand side and a polynomial in $\theta$ of degree 2 on the right hand side, both with positive leading coefficients. Therefore $\theta$ cannot be too large regarding $|\tau|$.

Finally, combining the conclusions of corollaries 1 and 2, we conclude that for any parameter set $(n, k, a, c)$ the value of $|-\tau-\theta|$ cannot be too big.

\section{Experimental Results}

In this section we present some experimental results for the admissibility conditions obtained in the previous section.

In Table 5.1 we consider the value $q_{\infty \alpha}^{2}$ from the right hand side of inequality (4.4) from Theorem 3 and we present the results for the parameter sets $P_{1}=(1275,364,63,120), P_{2}=(1296,435,90,174)$ and $P_{3}=$ $(1296,434,64,186)$ for different values of $\alpha$. For each set we present the respective eigenvalues $\theta$ and $\tau$.

\begin{tabular}{|c||c|c|c|}
\hline Parameters & $P_{1}$ & $P_{2}$ & $P_{3}$ \\
\hline \hline$\theta$ & 4 & 3 & 2 \\
\hline$\tau$ & -61 & -87 & -124 \\
\hline$q_{\infty 0.01}^{2}$ & $-2.0 \times 10^{-5}$ & $-2.2 \times 10^{-5}$ & $-2.3 \times 10^{-5}$ \\
\hline$q_{\infty 0.5}^{2}$ & $-1.0 \times 10^{-3}$ & $-1.1 \times 10^{-3}$ & $-1.1 \times 10^{-3}$ \\
\hline$q_{\infty 1}^{2}$ & $-1.9 \times 10^{-3}$ & $-2.2 \times 10^{-3}$ & $-2.3 \times 10^{-3}$ \\
\hline$q_{\infty 55}^{2}$ & $-9.5 \times 10^{-3}$ & $-1.1 \times 10^{-2}$ & $-1.1 \times 10^{-2}$ \\
\hline$q_{\infty 10}^{2}$ & $-1.9 \times 10^{-2}$ & $-2.2 \times 10^{-2}$ & $-2.2 \times 10^{-2}$ \\
\hline$q_{\infty 50}^{2}$ & $-7.3 \times 10^{-2}$ & $-1.1 \times 10^{-1}$ & $-1.1 \times 10^{-1}$ \\
\hline
\end{tabular}

Table 5.1: Numerical results for $P_{1}, P_{2}$ and $P_{3}$.

Next we present some examples of parameter sets $(n, k, a, c)$, with $k<$ $n / 2$, that do not verify the inequality (4.7) from Corollary 1 and the corresponding complement parameter sets $(n, \bar{k}, \bar{a}, \bar{c})$, with $k>n / 2-1$, that do not verify inequality (4.8) of Corollary 2. In Table 5.2 we consider the parameter sets $P_{4}=(1024,385,36,210), P_{5}=(1225,456,39,247)$, $P_{6}=(1296,481,40,260)$ and $P_{7}=(1275,378,57,135)$. For each set we 
present the respective eigenvalues $\theta$ and $\tau$, and the value

$\mathrm{m}_{1}=\tau(-2 \tau-1)(4 \theta-2 \tau+1)+\frac{2 n}{n-2(k-\theta)} \theta(\theta+1)(2 \theta-2 \tau-1)(\theta-\tau+1)$,

obtained from the inequality (4.7) of Corollary 1.

\begin{tabular}{|c||c|c|c|c|}
\hline Parameters & $P_{4}$ & $P_{5}$ & $P_{6}$ & $P_{7}$ \\
\hline \hline$\theta$ & 1 & 1 & 1 & 3 \\
\hline$\tau$ & -175 & -209 & -221 & -81 \\
\hline $\mathrm{m}_{1}$ & $-2.1 \times 10^{7}$ & $-3.5 \times 10^{7}$ & $-4.2 \times 10^{7}$ & $-1.45 \times 10^{6}$ \\
\hline
\end{tabular}

Table 5.2: Numerical results for $P_{4}, P_{5}, P_{6}$ and $P_{7}$.

From the data presented in Table 5.2 we confirm the results expressed in Corollary 1 , namely we confirm that if $\theta$ is much smaller than $|\tau|$, then we conclude that the sequence $(n, k, a, c)$ does not correspond to a parameter set of a strongly regular graph.

In Table 5.3 we present the respective complement parameter sets of $P_{1}$, $P_{2}$ and $P_{3}$, denoted by $\overline{P_{4}}=(1024,638,462,290), \overline{P_{5}}=(1225,768,558,352)$, $\overline{P_{6}}=(1296,814,592,374)$ and $\overline{P_{7}}=(1275,896,652,576)$. For each set we present the respective eigenvalues $\theta$ and $\tau$, and the value

$$
\begin{aligned}
\mathrm{m}_{2} & =\frac{2 n(-\tau+1)(-\tau+2)(-2 \tau+2 \theta-1)(\theta-\tau+1)}{2(k-\tau+2)-n} \\
& -(\theta-1)(2 \theta-3)(-4 \tau+2 \theta+3),
\end{aligned}
$$

obtained from the inequality (4.8) from Corollary 2.

\begin{tabular}{|c||c|c|c|c|}
\hline Parameters & $\overline{P_{4}}$ & $\overline{P_{5}}$ & $\overline{P_{6}}$ & $\overline{P_{7}}$ \\
\hline \hline$\theta$ & 174 & 208 & 220 & 80 \\
\hline$\tau$ & -2 & -2 & -2 & -4 \\
\hline $\mathrm{m}_{2}$ & $-1.6 \times 10^{7}$ & $-2.8 \times 10^{7}$ & $-3.4 \times 10^{7}$ & $-1.7 \times 10^{5}$ \\
\hline
\end{tabular}

Table 5.3: Numerical results for $\overline{P_{4}}, \overline{P_{5}}, \overline{P_{6}}$ and $\overline{P_{7}}$.

From the data presented in Table 5.3 we confirm the results expressed in Corollary 2, namely it is confirmed that if $|\tau|$ is much smaller than $\theta$, then the sequence $(n, k, a, c)$ does not correspond to a parameter set of a strongly regular graph.

Acknowledgment 1. 1. Enide Andrade Martins is supported in part by FEDER funds through COMPETE Operational Programme Fac- 
406 Vasco Moço M., Luís A. de Almeida V. and Enide Andrade M.

tors of Competitiveness ("Programa Operacional Factores de Competitividade") and by Portuguese funds through the Center for Research and Develpment in Mathematics and Applications and the Portuguese Foundation for Science and Technology ("FCT - Fundação para a Ciência e a Tecnologia") within project PEest-C/MAT/UI4106/ 2011 with COMPETE number FCOMP-01-0124-FEDER-022690 and Project PTDC/MAT/ 112276/2009.

2. Luís Vieira research funded by the European Regional Development Fund through the program COMPETE and by the Portuguese Government through the FCT - Fundação para a Ciência e a Tecnologia under the project PEest-C/MAT/UI0144/2011.

\section{References}

[1] L. W. Beineke, R. J. Wilson and P. J. Cameron, eds., Topics in Algebraic Graph Theory, Cambridge University Press, (2004).

[2] R. C. Bose, Strongly regular graphs, partial geometries and partially balanced designs, Pacific J. Math 13, pp. 389-419, (1963).

block designs with two associate classes, J. Am. Statist. Assoc. 47, pp. 151-184, (1952).

[3] A. E. Brouwer and J. H. van Lint, Strongly regular graphs and partial geometries, Enumeration and Design (D. M. Jackson and S. A. Vanstone, eds.), Academic Press, (1982).

[4] D. M. Cardoso and L. A. Vieira, Euclidean Jordan algebras with strongly regular graphs, Journal of Mathematical Sciences 120, pp. 881-894, (2004).

[5] Ph. Delsarte, J. M. Goethals and J. J. Seidel, Bounds for system of lines and Jacobi polynomials, Philips Res. Rep. 30, pp. 91-105, (1975).

[6] J. Faraut and A. Korányi, Analysis on Symmetric Cones, Oxford Science Publications, Oxford, (1994).

[7] L. Faybusovich, Euclidean Jordan algebras and interior-point algorithms, J. Positivity 1, pp. 331-357, (1997). 
[8] L. Faybusovich, Linear systems in Jordan algebras and primal-dual interior-point algorithms, Journal of Computational and Applied Mathematics 86, pp. 148-175, (1997).

[9] C. Godsil and G. Royle, Algebraic Graph Theory, Springer-Verlag, New York, (2001).

[10] R. Horn and C. R. Johnson, Matrix Analysis, Cambridge University Press, Cambridge, (1985).

Cambridge, (1991).

[11] X. L. Hubaut, Strongly regular graphs, Discrete Math. 13, pp. 357-381, (1975).

[12] P. Jordan, J. V. Neuman, and E. Wigner, On an algebraic generalization of the quantum mechanical formalism, Annals of Mathematics 35, pp. 29-64, (1934).

[13] M. Koecher, The Minnesota Notes on Jordan Algebras and Their Applications, Springer, Berlin, (1999).

[14] J. H. V. Lint and R. M. Wilson, A Course in Combinatorics, Cambridge University Press, Cambridge, (2004).

[15] V. M. Mano, E. A. Martins and L. A. Vieira, Feasibility conditions on the parameters of a strongly regular graph, Electronic Notes in Discrete Mathematics 38, pp. 607-613, (2011).

[16] V. M. Mano and L. Vieira, Admissibility conditions and asymptotic behavior of strongly regular graphs, International Journal of Mathematical Models and Methods in Applied Sciences, Issue 6, Vol 5, pp. 1027-1034, (2011).

[17] H. Massan and E. Neher, Estimation and testing for lattice conditional independence models on Euclidean Jordan algebras, Ann. Statist., 26, pp. 1051-1082, (1998).

[18] A. Neumaier, Strongly regular graphs with smallest eigenvalue -m, Archiv der Mathematik 33, pp. 392-400, (1979).

[19] L. L. Scott Jr., A condition on Higman's parameters, Notices of Amer. Math. Soc. 20 (1973) A-97 (Abstract 721-20-45). 
408 Vasco Moço M., Luís A. de Almeida V. and Enide Andrade M.

\author{
Vasco Moço Mano \\ Department of Mathematics \\ Faculty of Sciences \\ University of Porto, \\ Rua do Campo Alegre, \\ 687; 4169-007, Porto, \\ Portugal \\ e-mail : vascomocomano@gmail.com
}

\title{
Enide Andrade Martins
}

CIDMA - Center for Research and Development in Math. and Appl.

Department of Mathematics,

University of Aveiro,

3810-193 Aveiro,

Portugal

e-mail : enide@ua.pt

and

\section{Luís António de Almeida Vieira}

CMUP - Center of Research of Mathematics

Department of Mathematics

Faculty of Sciences

University of Porto,

Rua do Campo Alegre, 687; 4169-007, Porto,

Portugal

e-mail : lvieira@fe.up.pt 